\title{
Field Conjugation Adaptive Arrays in Atmospheric Coherent Optical Links
}

\author{
Aniceto Belmonte \\ Technical University of Catalonia, Department of Signal Theory and Communications, 08034 Barcelona, Spain \\ belmonte@tsc.upc.edu \\ Joseph M. Kahn \\ Stanford University, Department of Electrical Engineering, Stanford, CA 94305, USA \\ jmk@ee.stanford.edu
}

\begin{abstract}
We study the performance of field conjugation adaptive arrays applied in synchronous laser communication through the turbulent atmosphere. We assume that a single information-bearing signal is transmitted over the atmospheric fading channel, and that the adaptive array receiver combines multiple dependent replicas to improve detection efficiency. We consider the effects of log-normal amplitude fluctuations and Gaussian phase fluctuations, in addition to local oscillator shot noise. We study the effect of various parameters, including the ratio of receiver aperture diameter to wavefront coherence diameter, the scintillation index, and the number of branches combined at the receiver. We consider both maximal-ratio combining (MRC) and equal-gain combining (EGC) schemes.
\end{abstract}

Keywords-component; Atmospheric turbulence; Optical communications; Coherent receivers; Adaptive array receivers; Shannon capacity.

\section{INTRODUCTION}

Evaluating the performance of a heterodyne receiver in the presence of atmospheric turbulence is generally difficult because of the complex ways turbulence affects the coherence of the received signal that is to be mixed with the local oscillator (Fig. 1). Light propagated through a turbulent atmosphere contains speckle which will be present at the detector surface. Therefore, illuminating a single-element detector with a uniform LO beam will produce mismatch of the amplitudes and phases of the two fields resulting in a loss in downconverted power. The downconverted heterodyne power is maximized when the spatial field of the received signal matches that of the local oscillator $[1,2]$.

We have already modeled the impact of atmospheric turbulence-induced phase and amplitude fluctuations on freespace optical links using synchronous detection and found that the SNR $\gamma$ for a single-aperture coherent receiver is described by a noncentral chi-square probability distribution function (PDF) with two degrees of freedom [3]. The model leading to the chi-square PDF is based on the observation that the downconverted signal current can be characterized as the sum of many contributions from $N$ different coherent regions within the aperture (see Fig. 2). In this model, the signal is

The research of Aniceto Belmonte was partially funded by the Spanish Department of Science and Innovation MICINN Grant No. TEC 2009-10025. characterized as the sum of a constant (coherent) term and a random (incoherent) residual halo.

System configurations based on single apertures that are immune to atmospheric fluctuations are being developed [3,4]. The main goal is the improvement of the performance achievable in coherent, free-space optical communication systems using atmospheric compensation techniques. As an alternative to a large single-aperture coherent receiver, a large effective aperture can be achieved by combining the output signal from an array of smaller receivers (see Fig. 1). The advantage of a coherent array in terms of coupling efficiency is that the number of turbulence speckles over each subaperture in the array is much smaller than it would be over a single large aperture. Because each receiver can now be smaller than the scale on which the signal wavefront varies, the local oscillator phase can be matched to the signal to achieve effective coherent reception. The output signals from these receivers can be scaled and phase-shifted, and then combined electronically. Such electronic field conjugation can correct fluctuations of both amplitude and phase in the aperture plane, significantly improving detection statistics. In general, the performance of a field-conjugating system should improve with an increasing number of receivers for a fixed total aperture area.

We define a general model for the output SNR of diversity systems over correlated fading channels. For field conjugation adaptive arrays, where the atmospheric fading on the branches is correlated or dependent, we can solve the problem by transforming it into an independent problem using the technique of spatial whitening [5]. In this way, the results for independent fading channels [6] are easily extended to the more general problem of correlated channels. We have developed analytical expressions for the ergodic and outage capacities for free-space optical communication links using diversity combining from an information theory perspective [6].

\section{ARRAY BRANCHES COMBINING}

We consider two types of receive diversity combining [7]. First, we assume the receiver has knowledge of the instantaneous channel state, making perfect maximal ratio 
combining (MRC) diversity possible. In this diversity scheme, the receiver co-phases the intermediate signals, adjusts their amplitudes separately, and sums them to obtain a composite signal with improved SNR. The rate at which phase and amplitude must be adjusted will be dictated by the rate at which the atmospheric turbulence fluctuates, typically no higher than $1 \mathrm{kHz}$. The MRC receiver is the optimal combining technique in that it yields a carrier with the highest mean SNR and lowest SNR fading. The optimum electronic gain for each receiver should be proportional to the received signal field amplitude. Note that when the electronic gains and phase delays are back-propagated into the LO, the optimum gain and phase adjustments would result in an amplitude and phase match of this synthetic LO field to the distorted signal field.

Second, we consider the case when equal-gain combining (EGC) diversity is used. Since EGC diversity does not require estimation of the fading amplitudes, its complexity is smaller than the MRC approach. Although not optimal, EGC diversity is still an effective means of combating atmospheric fading. EGC diversity combining is equivalent mathematically to using adaptive optics to correct the distorted signal wave front to better match the LO wave front.

\section{OUTAGE SPECTRAL EFFICIENCY}

In this work, we analyze the spectral efficiency of optical communication over the clear turbulent atmosphere. Defined as the average transmitted data rate per unit bandwidth for a specific average transmit power and probability of outage or error, achievable spectral efficiency is an important performance measure for free-space optical communication systems. As the use of multilevel phase modulation schemes increase spectral efficiency by sending multiple bits per symbol, optical coherent reception provides an alternative to direct detection schemes for free-space optical communication applications. Certainly, both spectral efficiency and power efficiency need to be considered in the development of transmitter and receiver systems that extent the performance of free-space optical communication systems and provide suitable long-haul and space links. As we intend to focus on spectral efficiency, in this paper we do not address the receiver sensitivities in terms of photon efficiency. Figures 3, 4, and 5 show the results of this analysis.

Figures 3 and 4 consider the effect of aperture diameter on the $\varepsilon$-outage spectral efficiency. They present the spectral efficiency as a function of the normalized aperture $D / r_{0}$ for a constant phase coherence length $\mathrm{r}_{0}$. In Fig. 3, MRC combining is employed. In Fig. 4, a EGC combiner is considered. In all cases, the outage probability is small and fixed at $\varepsilon=0.001$, and the channel capacity per unit bandwidth is shown for different values of the number $L$ of combiner correlated branches. The case $L=1$ corresponds to no receive diversity. The area $\pi D^{2}$ describes the combined, array system equivalent aperture. When no receive diversity is considered, $D$ equals the receiver aperture diameter. If a $L$-aperture array is analyzed, each one of the aperture diameters equals $D / \sqrt{ } L$. For the smallest aperture considered, we assume $\gamma_{0}$ equal to 10 photons-per-symbol. For any other aperture diameter, the value of $\gamma_{0}$ is proportional to $D^{2}$. We study the outage channel capacity as a function of several parameters: the average signal-to-noise ratio (photons) per bit, the strength of atmospheric turbulence, the receiver aperture diameter $D$, and the number $L$ of combiner branches. Turbulence effects are described by two parameters. Fried's coherence length $r_{0}$ describes the coherent diameter of the distorted wavefront phase. The scintillation index $\sigma_{\beta}^{2}$ describes the intensity of amplitude fluctuations. In our modeling, we have chosen typical values of these two parameters.

When a single-receiver $(L=1)$ or dual-receiver $(L=2)$ system is considered, the existence of an optimal aperture diameter in coherent free-space links is apparent. This optimal aperture diameter maximizes the $\varepsilon$-outage capacity. When the aperture is larger than the optimal value, phase distortion cannot be overcome by the increase in collected power, and we observe a decrease in capacity. For systems using $L>2$ receivers, or under strong scintillation conditions, no optimal value can be identified.

Atmospheric fading leads to serious degradation in the receiver sensitivity measured in terms of received photons per symbol, resulting in either a higher error rate or a higher required transmit power for a given multilevel modulation technique. In general, the performance of an array signalcombining system should improve with an increasing number of receivers and, consequently, given a fixed collecting area, the combined system can offer superior performance. In Fig. 5, note the diminishing capacity returns that are obtained as the number of branches increases. Although as we increase $L$ the outage spectral efficiency improves appreciably, the greatest improvement is still obtained in going from single- to two- or four-branch combining.

\section{CONCLUSIONS}

The noncentral chi-square distribution, which we introduced recently as a model for atmospheric fading in a single coherent receiver affected by amplitude and phase fluctuations, is used to study the performance of coherent laser communication through the turbulent atmosphere. We have compared the spectral efficiencies and SNR requirements of complex modulations in the presence of fading noise from atmospheric turbulence and local oscillator shot noise. We have discussed methods of optimizing receiver sensitivity by using field conjugation adaptive arrays. We have identified the impact of the combiner number of branches on the performance of coherent modulation formats. For typical turbulence conditions, sizeable gains in spectral efficiency and SNR requirement are realizable by using a rather small number of sub-apertures.

More detailed results and comments on our analysis will be presented at the meeting.

\section{REFERENCES}

[1] D. L. Fried, "Optical heterodyne detection of an atmospherically distorted signal wave front," Proc. IEEE 55, 57-67 (1967)

[2] R. M. Gagliardi and S. Karp, Optical Communications (John Wiley \& Sons, 1995).

[3] A. Belmonte and J. M. Kahn, "Performance of synchronous optical receivers using atmospheric compensation techniques," Opt. Express 16, 14151-14162 (2008) 
[4] I. Kim, G. Goldfarb and G. Li, "Electronic wavefront correction for PSK free-space optical communications," Electron. Lett. 43, 1108-1109 (2007).

[5] S. Furrer, P. Coronel, and D. Darhlhaus, "Simple ergodic and outage capacity expressions for correlated diversity ricean fading channels," IEEE Trans. on Wireless Comm. 5, 1606-1609 (2006).
[6] A. Belmonte and J. M. Kahn, "Capacity of coherent free-space optical links using diversity-combining techniques," Opt. Express 17, 1260112611 (2009)

[7] J. D. Parsons, "Diversity techniques in communications receivers," in Advanced Signal Processing, D. A. Creasey, ed. (Peregrinus, 1985), Chap. 6.

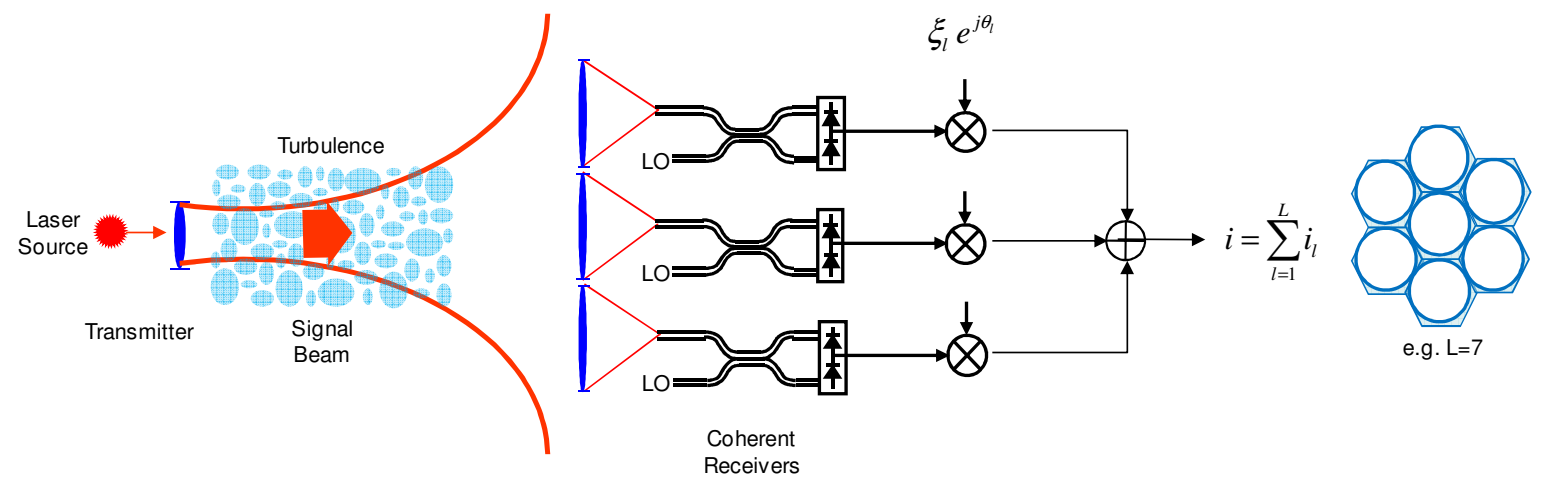

Figure 1. A coherent free-space optical communication system is affected by the presence of atmospheric turbulence in several ways. First, turbulence induces a decrease of the mean received power level, which translates into a diminished signal-to-noise ratio in the receiver. Second, amplitude scintillation and phase distortion in the receiver plane act as intense sources of noise distorting the quality of the optical signal available for processing. These add together to deteriorate the overall communication performance of the optical systems. Adaptive arrays could be considered to alleviate the deteriorating effects of atmospheric turbulence on the performance of coherent receivers in free-space optical communications. 


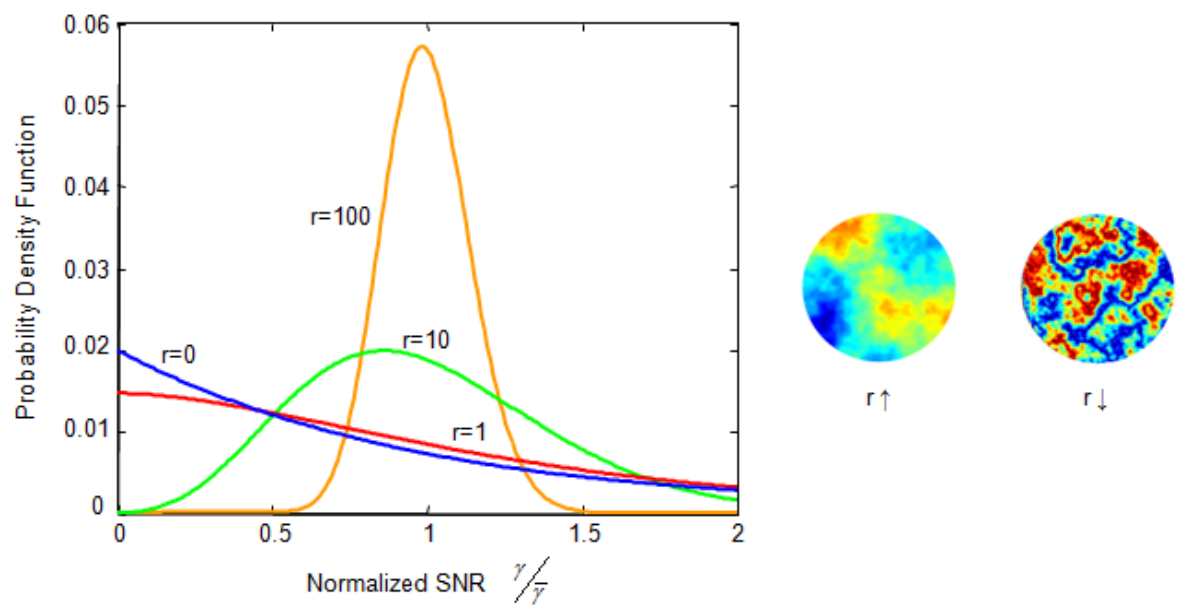

Figure 2. By noting that the downconverted signal current can be characterized as the sum of many contributions from different coherent regions within the aperture, we show that the probability density function (PDF) of this current can be well-approximated by a noncentral chisquare probability distribution [3]. We show the density function as a function of the normalized SNR and the Rician factor $r$.

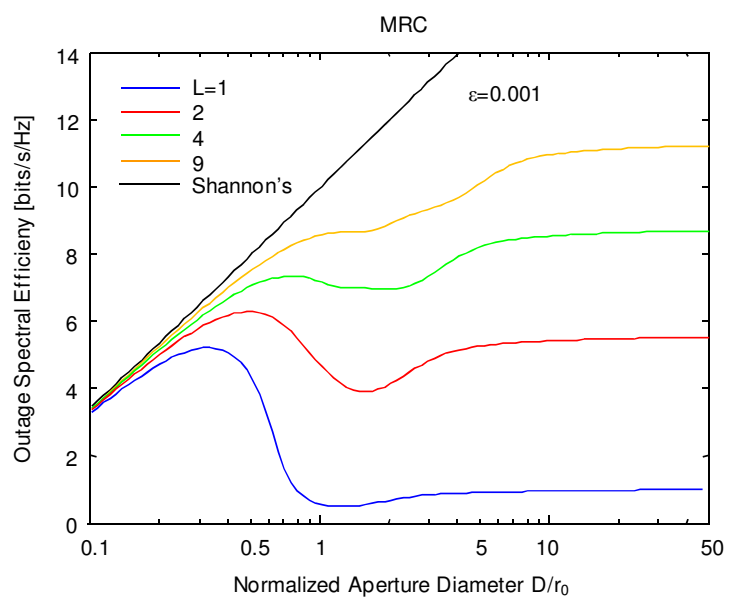

Figure 3. $\varepsilon$-outage spectral efficiency vs. normalized receiver aperture diameter $D / r_{0}$ for coherent detection and AWGN. MRC combining is employed. In all cases, the outage probability is fixed at $\varepsilon=0.001$, and the channel capacity per unit bandwidth is shown for different values of the number $L$ of combiner branches. The case $L=1$ corresponds to no receive diversity (blue lines). The area $\pi D^{2}$ describes the combined, array system equivalent aperture. The turbulence-free SNR per symbol $\gamma_{0}$ is proportional to the square of the aperture diameter $D$. For the smallest aperture considered, we assume $\gamma_{0}$ equal to 10 photons per symbol. In this plot, we neglect amplitude fluctuations by assuming $\sigma_{\beta}{ }^{2}=0$. In this case, turbulence is characterized by the phase coherence length $r_{0}$. The AWGN Shannon limit is indicated by the black line. 


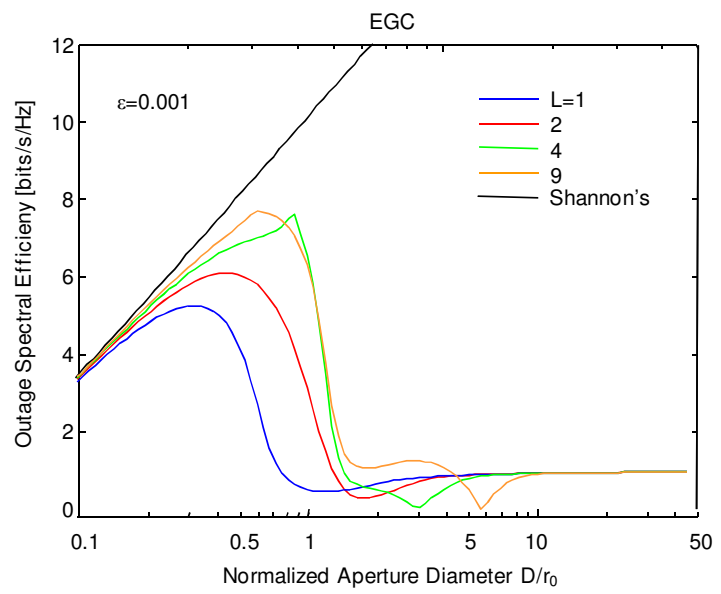

Figure 4. $\varepsilon$-outage spectral efficiency vs. turbulence-free photons per symbol $\gamma_{0}$ for coherent detection and additive white Gaussian noise (AWGN). An EGC combiner is considered. All other parameters are as in Fig. 3.

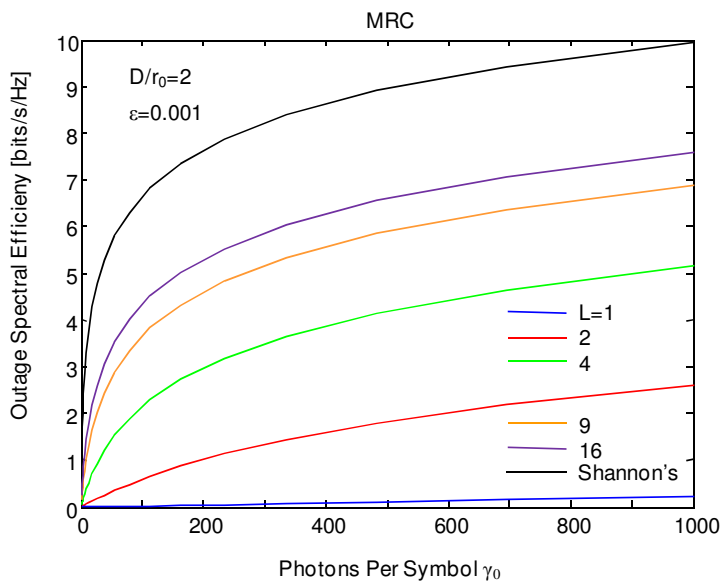

Figure 5. $\varepsilon$-outage spectral efficiency vs. turbulence-free photons per symbol $\gamma_{0}$ for coherent detection and additive white Gaussian noise (AWGN). MRC combining is employed. In all cases, the outage probability is fixed at $\varepsilon=0.001$, and the channel capacity per unit bandwidth is shown for different values of the number $L$ of correlated combiner branches. The case $L=1$ corresponds to no receive diversity (blue lines). Amplitude fluctuations are neglected by assuming $\sigma_{\beta}{ }^{2}=0$. Turbulence is characterized by a moderate phase coherence length $r_{0}$ such as $D / r_{0}=2$. The area $\pi D^{2}$ describes the combined, array system equivalent aperture. The AWGN Shannon limit is indicated by black lines. 